\title{
Origin of the pulsar pulse fine structure
}

\section{O. M. Ulyanov, A. A. Seredkina and A. I. Shevtsova}

Department of Astrophysics, Institute of Radio Astronomy of NAS of Ukraine, Krasnoznamennaya str.4, Kharkov 61002, Ukraine

email: oulyanov@rian.kharkov.ua, seredkina.a@gmail.com, alice.shevtsova@gmail.com

\begin{abstract}
We give a new numerical model of pulsar pulse radiation through the interstellar medium (ISM) considering the propagation effects. It explains the deficit of a scattering measure at the decameter range of frequencies that leads to the possibility of detecting the pulsar pulse fine structure. The results of numerical simulation confirm that the fine structure may be detected at low frequencies and this is qualitatively agreed with the observational data.
\end{abstract}

Keywords. interstellar medium (ISM), scattering, pulsar.

\section{Introduction}

Despite the fact that the micro structure of pulsar radio emission was discovered more than 40 years ago (Hankins 1971) still there is no generally accepted model of an origin of this phenomenon. We consider a conception of a fine structure of pulsar radio emission that covers different time scales from nanoseconds in the centimeter wave range to milliseconds in the decameter wave range. We propose a new model of the fine structure formation that is considered as a result of the propagation of the radio pulses through the interstellar medium and subsequent processing of the received signal in the lab frame.

\section{Observational Data}

The model gives us the value of the scattering time constant $\tau_{s c}(f)$ at the frequency $f$ that describes the temporal broadening of pulses due to interstellar scattering: $\tau_{s c}(f)=$ $\tau_{s c, 0}\left(f_{0}\right)\left(f / f_{0}\right)^{-\alpha}$, where $\tau_{s c, 0}\left(f_{0}\right)$ is the scattering time constant at the fixed frequency $f_{0}$ that one could take from a pulsar catalogue (usually $f_{0}=1 \mathrm{GHz}$ ); $\alpha$ is a power that corresponds to different spectra of spatial inhomogeneities of the electron concentration.
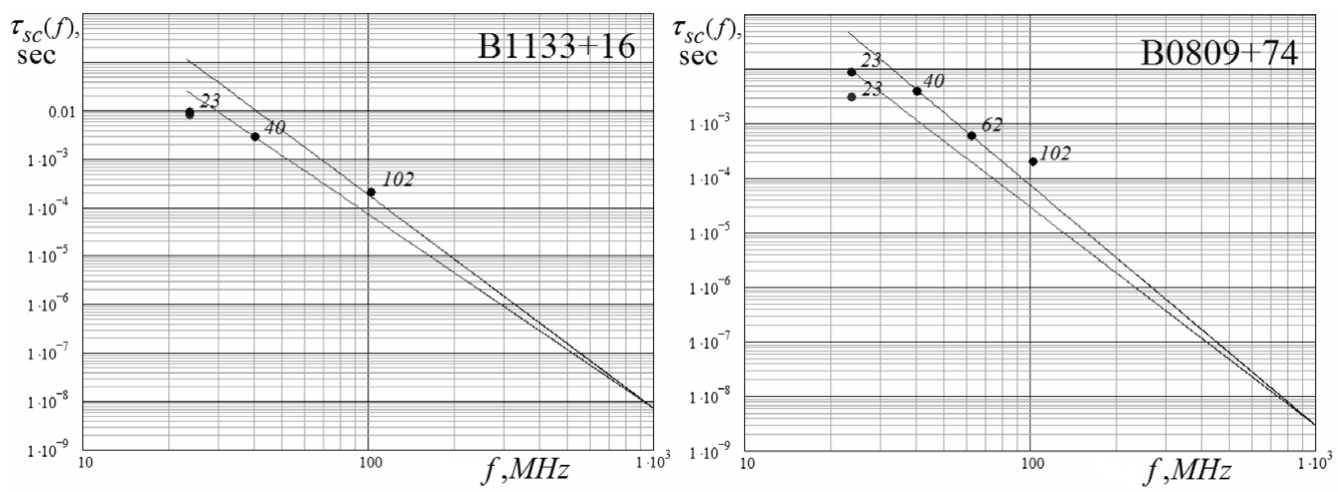

Figure 1. Scattering time constant at different frequencies. The normal spectrum of electron inhomogeneities (upper line); Kolmogorov spectrum of electron inhomogeneities (lower line). 
Thus $\alpha=4.0$ for the normal spectrum of the electron density fluctuations, $\alpha=4.4$ of the Kolmogorov spectrum.

The observation in the decameter range (Ul'yanov \& Zakharenko 2012) gives us the lower value of the scattering measure for anomalous-intensity pulses (Fig. 1) . It means that the modern model of the radio pulses propagation through the ISM is not completely correct. On the other hand, we are able to detect the fine structure of the pulsar pulses in the low frequency range that gives us more possibilities to study this phenomenon. The purpose of the present work is to create a model of ISM to explain the deficit of a scattering measure and formation of the fine structure of the pulsar radio emission.

\section{Numerical Simulation}

The short radio pulses have been generated in a wide frequency range with the pulsar rotation period $\mathrm{P}=1 \mathrm{sec}$. The pulses are represented as an amplitude modulated noise signal that has a Gauss shape envelope. The width of the envelope is about $10 \%$ of the pulses period. The signal has the uniform distribution of the frequencies in the receiving range $\Delta f=f_{H}-f_{L}$ and the uniform distribution of the initial phases in the angles range $[-\pi, \pi]$. The noise signal amplitudes have the Rayleigh distribution. Also the white noise with the normal distribution is added to the propagation channel.

The main idea of the present paper is to create an ISM model that describes the scattering measure deficit. We are taking into account two main factors: the dispersion delay of low frequencies versus high frequencies and the scattering by space irregularities of the electron concentration. We do not consider the Faraday rotation effect.

The dispersion delay of low frequencies versus high frequencies is the result of the relationshep between the refraction index and frequency in the cold anisotropic ISM plasma. Using the eikonal equation we write the delay phase $\varphi(\omega)$ :

$$
\varphi(\omega) \approx \omega \frac{L}{c}-\frac{1}{\omega} \frac{2 \pi e^{2}}{m_{e} c} \int_{0}^{L} N_{e}(z) d z,
$$

where $\omega$ is a cyclic frequency; $e, m_{e}$ are the electron charge and its rest mass; $c$ is the speed of light. $D M=\int_{0}^{L} N_{e}(z) d z$ is the dispersion measure, which characterizes the number of free electrons along the line of sight and is measured in units of $\mathrm{pc} \cdot \mathrm{cm}^{-3}$.

We can also define the time delay of a frequency $\omega_{i}$ versus infinity to be able to reduce the dispersion in the received signal . From the equation (3.1): $\Delta \tau_{D M}\left(D M, \omega_{i}\right)=$ $\frac{2 \pi e^{2}}{m_{e} c} D M\left(\frac{1}{\omega_{i}}\right)^{2}$

We also consider the scattering of the pulsar radiation by fluctuations in the concentration of free electrons in the propagation medium along the line of sight. These lead to the amplitude and phase fluctuations of the received wave, called scintillations. To describe the scintillations we use the thin screen model. In this model we consider only phase fluctuations of the original signal that occur in the thin screen that is perpendicular to the line of sight, located halfway the source and observer. The distance between the screen and the observer or the screen and the source must be much greater than the signal wavelength. In this case one could use the eikonal equation. Scattering on the thin screen leads to multibeam interference at the receiver and the point source becomes a finite extended object with angular size equal to the scattering angle $\theta_{s c}$.

The geometric optic approximation gives the scattering angle as a function $\theta_{s c}(\lambda)$ of wavelength: $\theta_{s c}(\lambda) \approx \frac{\Delta \varphi(\lambda)}{2 \pi} \frac{\lambda}{a}=\frac{r_{e} \delta N_{e} \lambda^{2}}{2 \pi}\left(\frac{D W}{a}\right)^{1 / 2}$, where $\lambda$ is the wavelength, $r_{e}$ is the electron radius, $\delta N_{e}$ is the fluctuation of the electron concentration, $a$ is the characteristic length scale of the electron-concentration irregularities, and $D W$ is the screen width. 

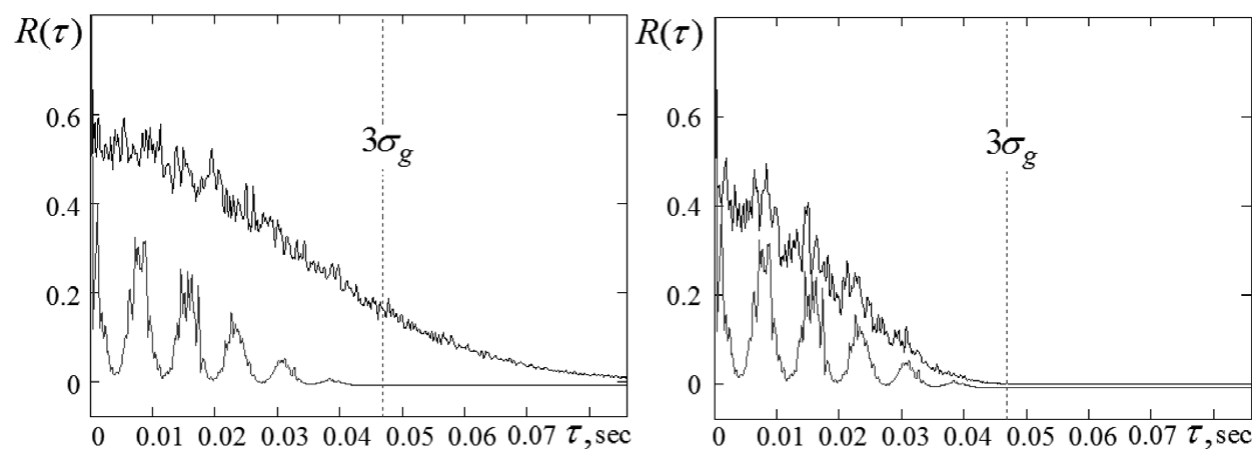

Figure 2. Input (lower) and output (upper) ACFs of the single pulses with the fine structure.

Large-scale and short-scale irregularities are given on the left and right sides respectively.

At the receiver the signal passes through a preselector filter. Then, we used the method of dispersion delay removing proposed by Hankins \& Rickett (1975). The method uses the eikonal equation to find the argument in the transfer function of the ISM. For the narrow band signal this argument $\Delta \alpha_{T}(\omega)$ can be expanded in a Taylor series centered at the receiver central frequency $f_{0}$. The initial phase in the observational reference frame can be written as: $\Delta \alpha_{T}(\omega)=\frac{2 \pi e^{2}}{m_{e} c} D M\left(\frac{1}{\omega_{i}}-\frac{\Delta \omega}{\omega_{i}{ }^{2}}+\frac{\Delta \omega^{2}}{\omega_{i}^{3}}-\frac{\Delta \omega^{3}}{\omega_{i}{ }^{4}}+\ldots\right)$

\section{Main Results}

As a result of the numerical simulation we obtained signal responses at different stages of the modeling. Then we studied the signals by using ACF and spectral analysis. At the sky frequency $f_{0}=20 \mathrm{MHz}$ the fine structure of the model pulse may be or may not be detected with different parameters of the ISM. The main parameter is the spatial irregularities size of the electron density. The data analysis results are shown in Fig. 2. It is qualitatively similar to real observational data†.

\section{Conclusions}

1) The presence or absence of the fine structure in the low frequency range is explained by a reaction of radio waves on the propagation in a plasma environment, which is located in the line of sight.

2) The fine structure is smoothed out more strongly by scattering at the large-scale spatial electron density irregularities, at the same time one can detect the fine structure of the pulsar radiation in case of scattering by small-scale irregularities.

3) The characteristic width of the detected fine structure is frequency dependent and increases with decreasing of the radiation frequency.

\section{References}

Hankins, T. H., 1971, ApJ, 169, 487

Hankins T. H. \& Rickett B. J. 1975, in: Methods in computational physics, Academic Press, Inc., 14,55

Ul'yanov, O. M. \& Zakharenko, V. V., 2012, Astr. Rep., vol. 56, 417

$\dagger$ For more details see

WwW.pulsarastronomy.net/IAUS291/download/Posters/IAUS291_Ulyanov0_257.pdf 